\title{
Correlations between nuclear morphology and bundles of cytoplasmic fibrils in 50 cases of acute myeloid leukaemia
}

\author{
EC PEARSON
}

From the Department of Haematological Medicine, University Clinical School, Hills Road, Cambridge

SUMMARY An electron microscopic examination was carried out of peripheral blood or bone marrow samples, or both, from 50 patients entered into the Medical Research Council 9th Acute Myeloid Leukaemia Trial. The results showed a striking correlation between the presence of conspicuous bundles of fibrils within the cytoplasm of the leukaemic cells and the degree of convolution or lobulation of the nuclei. In none of the samples were predominantly convoluted or lobed nuclei observed in the absence of prominent fibrillar bundles and in only two cases were nuclei of a more regular outline seen in association with many conspicuous bundles of cytoplasmic fibrils. No correlation was found between the apparent degree of maturity of the nuclei, as assessed by the degree of chromatin condensation, and the absence or abundance of fibrillar bundles.

The presence of prominent bundles of cytoplasmic fibrils in leukaemic cells, especially those of the monocyte series, has been recognised for some time. ${ }^{1-3} \mathrm{An}$ ultrastructural study of 50 patients entered for the Medical Research Council 9th Acute Myeloid Leukaemia Trial was carried out recently, and during the course of this study a strong correlation between the presence of prominent bundles of cytoplasmic fibrils and convoluted or lobed nuclei was observed. In this paper I present the data which led to this correlation and put forward a possible explanation. The data were also examined with a view to determining any correlation between the degree of maturation of the nuclei and the presence of fibrillar bundles in the cytoplasm.

\section{Material and methods}

Samples of peripheral blood (33 cases), bone marrow (14 cases), or both (three cases) were obtained before treatment from 50 patients entered for the trial. The 50 patients were classified as follows: acute myeloblastic leukaemia (FAB classification $\mathrm{M} 1 / \mathrm{M} 2)^{4}, 23$ cases; acute promyelocytic leukaemia (M3), five cases; acute myelomonocytic leukaemia (M4), 16 cases; acute monocytic leukaemia (M5), four cases; and mixed, two cases. The cases classified as mixed were those which showed strong diagnostic features of more than one of the preceding categories. A

Accepted for publication 2 September 1985 more extensive description of the ultrastructural features observed in the five cases of acute promyelocytic leukaemia included here has been presented previously as part of a larger study of ultrastructure and cytogenetics in acute promyelocytic leukaemia. ${ }^{5}$

Samples were prepared for electron microscopy using standard techniques. ${ }^{2}$ Ultrathin sections of material embedded in Spurr's resin ${ }^{6}$ were stained in uranyl acetate and Reynolds' lead citrate ${ }^{7}$ and observed in a Zeiss EM109 electron microscope. The presence of fibrillar bundles and convoluted nuclei was noted directly as the sections were observed in the electron microscope. Several sections were observed for each case, so that for each patient several hundred leukaemic cells were included in the analysis.

\section{Results}

During the course of the ultrastructural examination of the 50 cases of acute myeloid leukaemia determinations were made as to whether the nuclei of the leukaemic cells were predominantly convoluted or lobed and as to whether many, few, or no cells contained conspicuous bundles of fibrils within their cytoplasm. Table 1 shows the data for each of the five classes of acute myeloid leukaemia. Fig. 1 shows a blast cell from a case of acute myelomonocytic leukaemia showing a conspicuous bundle of cytoplasmic fibrils and part of a convoluted nucleus. In this, as in many similar cells, a prominent bundle of fibrils was present, but fibrils were not always obvious within the 
Table 1 Occurrence of prominent bundles of cytoplasmic fibrils and convoluted or lobed nuclei in 50 cases of acute myeloid leukaemia

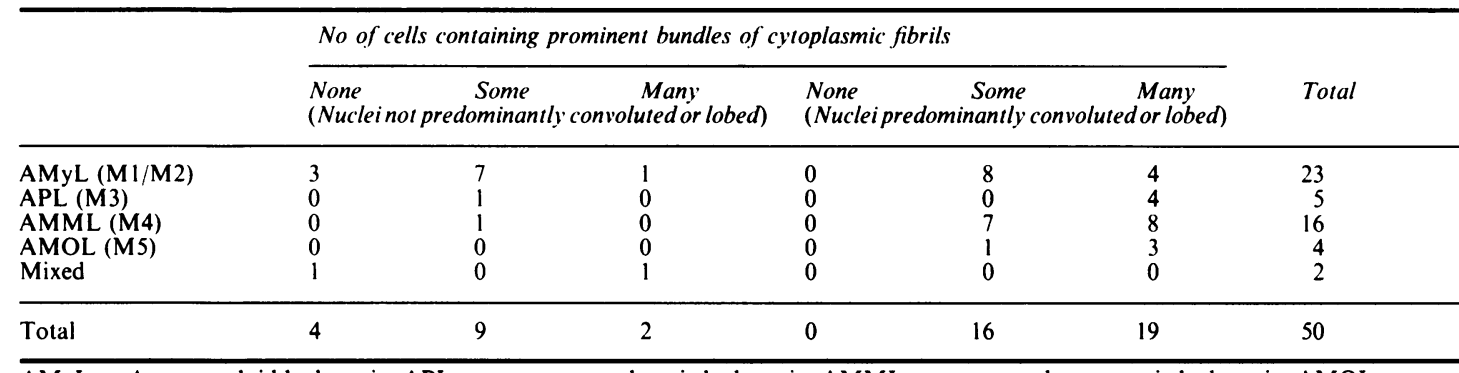

$\mathrm{AMyL}=$ Acute myeloid leukaemia; $\mathrm{APL}=$ acute promyelocytic leukaemia; $\mathrm{AMML}=$ acute myelomonocytic leukaemia; $\mathrm{AMOL}=$ acute monocytic leukaemia.

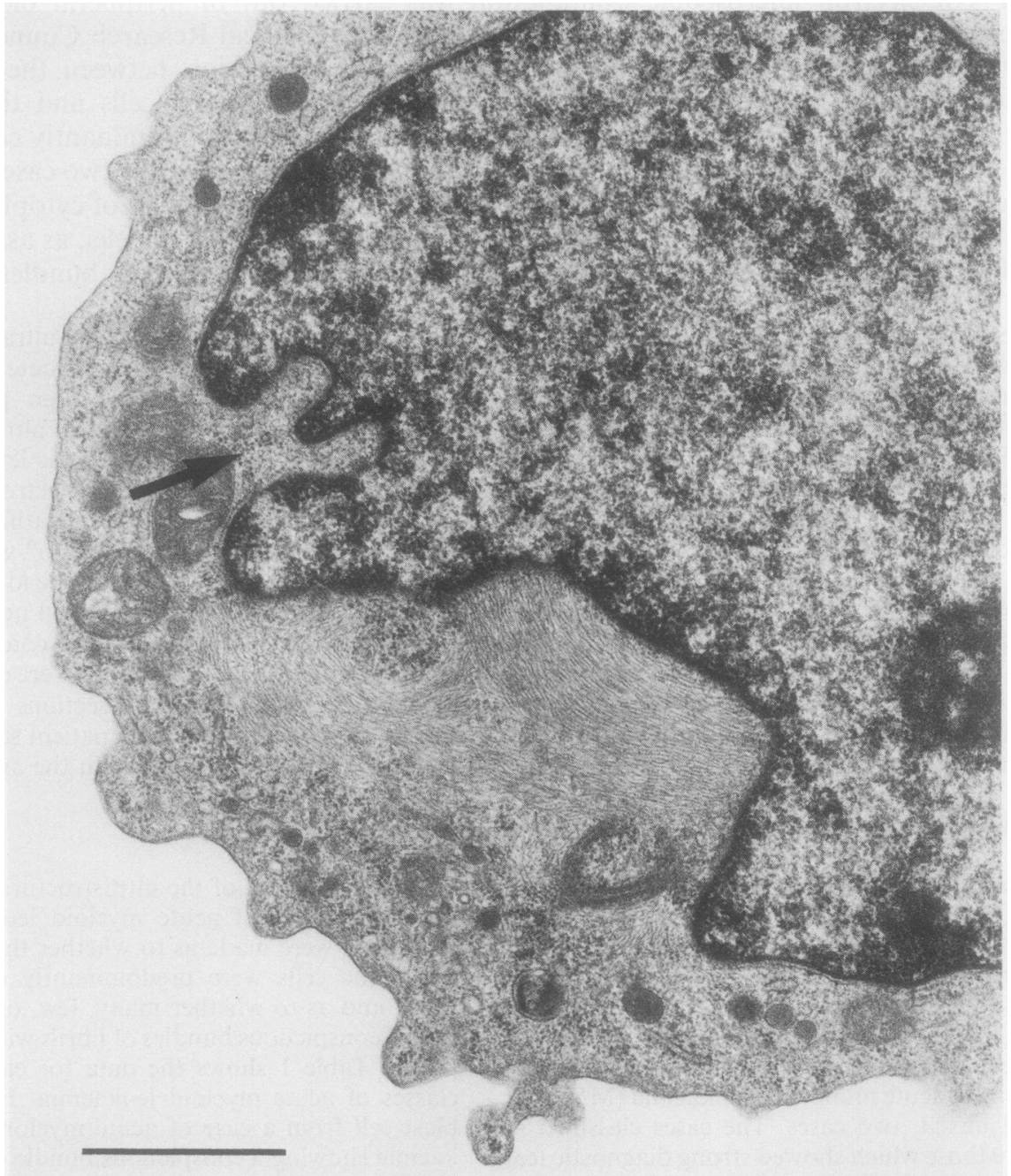

Fig. 1 Part of blast cell from peripheral blood of a case of acute myelomonocytic leukaemia. Conspicuous bundle of fibrils has apparently caused a large deformation of nuclear outline but no fibrillar bundles are visible within smaller indentations (arrow). Scale bar $=1 \mu \mathrm{m}$. 


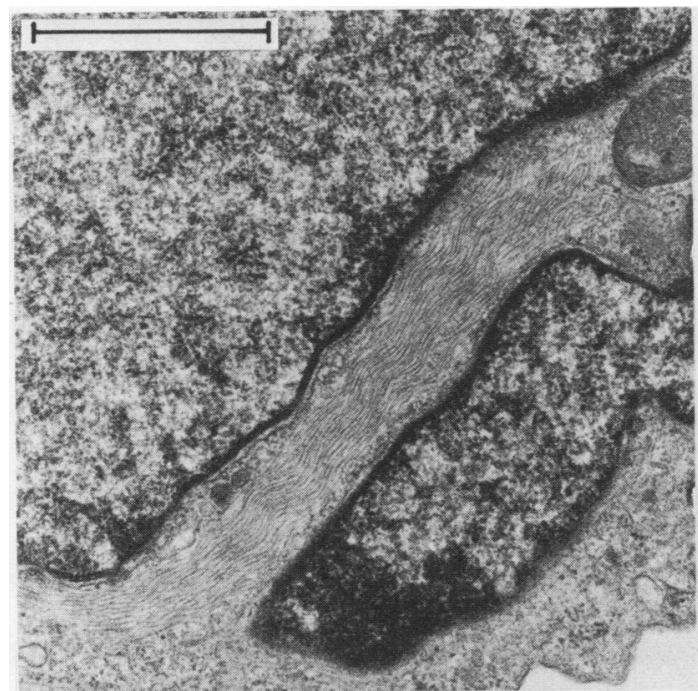

Fig. 2 Part of another blast cell from same case as Fig. 1. Bundle of fibrils in longitudinal section is visible between two lobes of nucleus, which was classified as showing a low degree of chromatin condensation. Scale bar $=1 \mu \mathrm{m}$. nuclear clefts. Figs. 2 and 3, however, show bundles of cytoplasmic fibrils contained within nuclear clefts or indentations. Occasionally, large bundles of fibrils seemed to be responsible for gentler deformations of the nuclear outline than the more abrupt indentations or clefts. Fig. 4 shows a concave depression in the nuclear membrane associated with a large bundle of cytoplasmic fibrils.

It was helpful to categorise the 50 cases of acute myeloid leukaemia, not on the basis of type of disease (Table 1), but simply on the basis of whether many, few, or no cells contained prominent bundles of cytoplasmic fibrils and then to consider what proportion of cells in each of the three categories contained nuclei which were predominantly convoluted or lobed (Table 2).

In addition, the degree of chromatin condensation within the nuclei was compared with the occurrence of bundles of cytoplasmic fibrils (Table 3). The degree of chromatin condensation, classified here simply as high (as in a normal mature granulocyte), intermediate (Fig. 5), or low (Fig. 2), is often regarded as an indication of the maturity of the nucleus. On this basis the
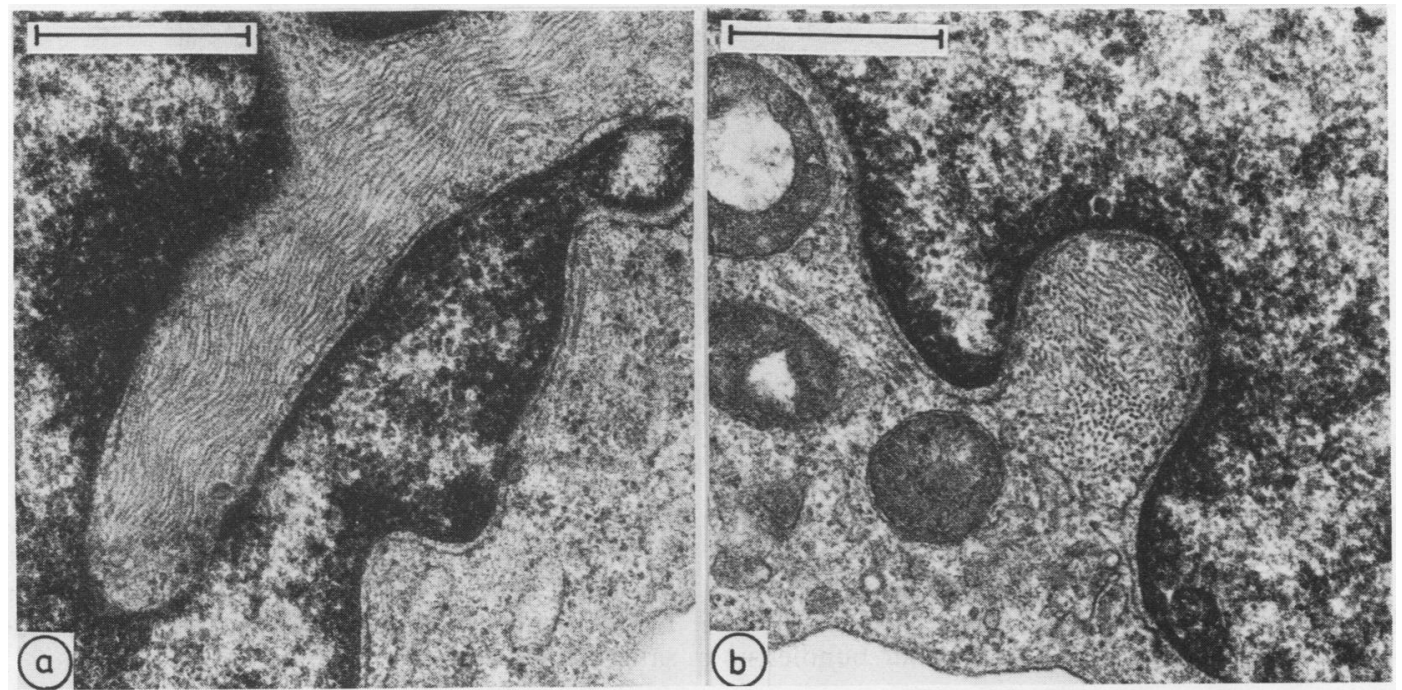

Fig. 3 Part of two blast cells from same case of acute myelomonocytic leukaemia as Fig. 1 showing (a) longitudinal and (b) transverse sections of bundles of cytoplasmic fibrils contained within nuclear indentations. Scale bar $=500 \mathrm{~nm}$.

Table 2 Fifty cases of acute myeloid leukaemia classified into groups having no, some, or many prominent bundles of cytoplasmic fibrils: the proportion of cases having predominantly convoluted or lobed nuclei in each category

\begin{tabular}{llccc}
\hline State of nuclei & \multicolumn{2}{l}{ No $(\%)$ of cells containing prominent bundles of cytoplasmic fibrils: } \\
\cline { 2 - 4 } & None & Some & Many & Total \\
\hline $\begin{array}{llcl}\text { Predominantly lobed or convoluted } \\
\text { Not predominantly lobed or convoluted }\end{array}$ & $\begin{array}{l}0 \\
4(100)\end{array}$ & $\begin{array}{c}16(64) \\
9(36)\end{array}$ & $\begin{array}{r}19(90) \\
2(10)\end{array}$ & $\begin{array}{l}35(70) \\
15(30)\end{array}$ \\
\hline Total & $4(100)$ & $25(100)$ & $21(100)$ & $50(100)$ \\
\hline
\end{tabular}


Fig. 4 Concave depression in nucleus apparently caused by large "swirling" bundle of fibrils contained within cytoplasm of blast cell from same case as Fig. 1 . Scale bar $=500 \mathrm{~nm}$.

data showed no correlation between the presence of fibrillar bundles and the degree of maturation of the nuclei. This was true whether the data were considered all together (Table 3) or after classification according to the type of disease (data not shown). The distribution (Table 3), of the number of fibrillar bundles in those cases predominantly showing a low degree of chromatin condensation was virtually identical with the distribution in those cases showing an intermediate degree of condensation. The cases predominantly showing a high degree of chromatin condensation were too few to analyse.

Table 3 Degree of chromatin condensation and occurrence of prominent bundles of cytoplasmic fibrils in 50 cases of acute myeloid leukaemia

\begin{tabular}{llcll}
\hline $\begin{array}{l}\text { No of cells containing } \\
\text { prominent bundles of } \\
\text { cytoplasmic fibrils }\end{array}$ & \multicolumn{2}{c}{ Degree (\%) of chromatin condensation } & High* & Total \\
\cline { 2 - 5 } & Low & Intermediate & $4(8)$ \\
None & $2(11)$ & $2(7)$ & 1 & $25(50)$ \\
Some & $9(47)$ & $15(52)$ & 1 & $21(42)$ \\
Many & $8(42)$ & $12(41)$ & 2 & $50(100)$ \\
\hline Total & $19(100)$ & $29(100)$ & \\
\hline
\end{tabular}

*Too few examples to calculate meaningful percentages. 


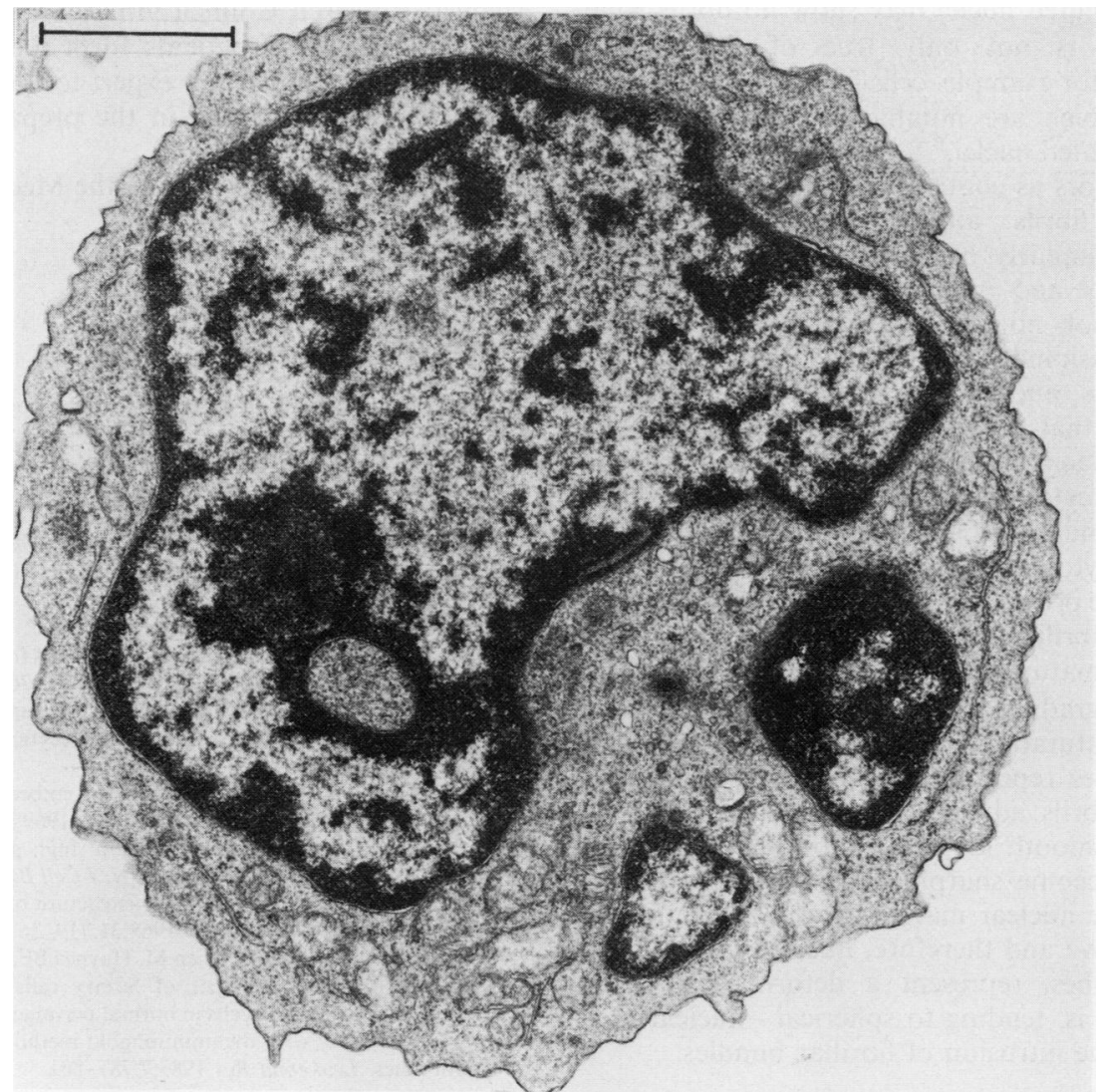

Fig. 5 Blast cell from peripheral blood of case of acute myeloblastic leukaemia. Although nucleus is lobed, there are no conspicuous bundles of cytoplasmic fibrils visible in the cell. Distribution of condensed chromatin around nuclear membrane and in patches within nuclear matrix is typical of "intermediate" classification of chromatin condensation. Scale bar $=1 \mu \mathrm{m}$.

\section{Discussion}

It is evident from the data (Tables 1 and 2) that there is a strong correlation between the presence of conspicuous bundles of cytoplasmic fibrils and convoluted or lobed nuclei in the 50 cases of acute myeloid leukaemia that formed the basis of this study. Conversely, in cases in which fibrillar bundles were not conspicuous the nuclei tended to be of a more regular outline. No cases showed predominantly convoluted of lobed nuclei in the absence of prominent fibrillar bundles, and in only two cases (two of 50) were nuclei of a more regular outline observed in the presence of many bundles of cytoplasmic fibrils.

Convoluted nuclei with associated bundles of cytoplasmic fibrils were almost invariably observed in acute myelomonocytic leukaemia (15 of 16) and acute monocytic leukaemia (four of four). In addition, as indicated previously, ${ }^{5}$ these features are also common in acute promyelocytic leukaemia, (four of five in this study; five of seven in our previous study, which included two additional cases not entered for the trial).

Of the 23 cases of acute myeloblastic leukaemia, 11 (11 of 23) did not have predominantly convoluted or lobed nuclei, although in one case there were many prominent bundles of fibrils. Of the 15 cases with nuclei of a more regular outline (15 of 50), most were those of acute myeloblastic leukaemia (11 of 15), two cases (two of 15) were of mixed leukaemias and the remaining two cases were one each of acute promyelocytic leukaemia and acute myelomonocytic leukaemia.

It would be incorrect to suggest that nuclear convolution or lobulation is necessarily always caused by the presence of fibrillar bundles, because by no means all nuclear clefts contained such bundles (Figs. 1 and 5), and, indeed, sections through leukaemic cells con- 
taining convoluted nuclei may show no fibrils whatsoever. This is not only true of the myeloid leukaemias - for example, cells from cases of Sézary's syndrome, which are notable for the presence of highly convoluted nuclei, ${ }^{8}$ have not been regarded by previous authors as containing excessive amounts of cytoplasmic fibrils although some have been observed. ${ }^{19}$ Similarly, normal mature leucocytes such as neutrophils and monocytes, which have lobed nuclei, are not noted for their fibrillar bundles, although occasionally they may be present. ${ }^{12}$

Nevertheless, nuclear convolution may, at least in cases such as that shown in Figs. 1-3, arise in acute myeloid leukaemia as a consequence of overproduction of cytoplasmic fibrils, which congregate to form large bundles and simply by their physical presence in the cytoplasm deform the nuclei. The data presented here provide no evidence to suggest that the incidence of fibrillar bundles increases (or decreases) with nuclear maturity (Table 3 ). This disagrees with the idea of a gradual accumulation (or loss) of fibrils during the maturation process of the leukaemic cell. In the 50 cases reported here the presence of large bundles of fibrils adjacent to the nuclei was often observed to smooth the nuclear outline rather than cause it to become sharply indented (Fig. 4). Such regions of the nuclear margin, however, are usually slightly concave and therefore, like the formation of clefts and lobes, represent a deformation of the normal-that is, tending to spherical-nuclear morphology by the intrusion of fibrillar bundles.

I thank Professor FGJ Hayhoe for critical comments on the manuscript and for continued encouragement, the clinicians throughout Britain participating in the
Medical Research Council 9th Acute Myeloid Leukaemia Trial whose patients form the basis of this study, Miss W Gillson for expert technical help, and Mrs J Chandler for help in the preparation of the manuscript.

This study was supported by the Medical Research Council.

\section{References}

${ }^{1}$ Tanaka Y, Goodman JR. Electron microscopy of human blood cells. New York: Harper and Row, 1972.

${ }^{2}$ Cawley JC, Hayhoe FGJ. Ultrastructure of haemic cells. London: WB Saunders Company Ltd, 1973.

${ }^{3}$ Breton-Gorius J, Gourdin MF, Reyes F. Ultrastructure of the leukemic cell. In: Catovsky D, ed. Methods in hematology, Vol. 2 The leukemic cell. Edinburgh: Churchil Livingstone, 1981: 87-128.

${ }^{4}$ Bennett JM, Catovsky D, Daniel M-T, et al. French-AmericanBritish (FAB) cooperative group. Proposals for the classification of the acute leukaemias. Br J Haematol 1976;33:451-8.

${ }^{5}$ Pearson EC, Matthews JG, Hayhoe FGJ. Ultrastructure and cytogenetics in seven cases of acute promyelocytic leukaemia (APL). Br J Haematol (in press).

${ }^{6}$ Spurr AR. A low viscosity epoxy resin embedding medium for electron microscopy. J Ultrastruct Res 1969;26:31-43.

${ }^{7}$ Reynolds ES. The use of lead citrate at high $\mathrm{pH}$ as an electronopaque stain in electron microscopy. J Cell Biol 1963;17:208-12.

${ }^{8}$ Lutzner MA, Jordan HW. The ultrastructure of an abnormal cell in Sézary's syndrome. Blood 1968;31:719-26.

${ }^{9}$ Matutes E, Robinson D, O'Brien M, Haynes BF, Zola H, Catovsky D. Candidate counterparts of Sézary cells and adult T-cell lymphoma-leukaemia cells in normal peripheral blood: an ultrastructural study with the immunogold method and monoclonal antibodies. Leukemia Res 1983;7:787-801.

Requests for reprints to: Dr EC Pearson, Department of Haematological Medicine, University Clinical School, Hills Road, Cambridge CB2 2QL, England. 